\title{
Estresse e ansiedade em mulheres inférteis
}

\author{
Stress and anxiety in infertile women

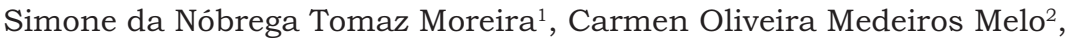 \\ Geraldez Tomaz ${ }^{3}$, George Dantas de Azevedo ${ }^{4}$
}

RESUMO

Objetivo: avaliar a freqüência de estresse e níveis de ansiedade em mulheres inférteis, de forma a obter subsídios para uma orientação psicológica específica. Métodos: estudo transversal envolvendo 152 mulheres inférteis (média de idade $30,3 \pm 5,4$ anos), e 150 controles saudáveis $(25,7 \pm 7,9$ anos). Todas as voluntárias foram avaliadas com a aplicação do Inventário de Sintomas de Estresse de Lipp e Inventário de Ansiedade Traço-Estado. As variáveis dependentes foram: freqüência de estresse e escores de ansiedade (estado e traço). A análise estatística compreendeu a utilização dos testes $\chi^{2} \mathrm{e}$ Mann-Whitney, além de regressão logística para testar associações entre as variáveis dependentes e fatores de risco considerados. O nível de significância adotado foi de 5\%. Resultados: a freqüência de estresse foi maior no grupo infertilidade em relação ao grupo controle ( 61,8 e 36,0\%, respectivamente), no entanto, não foram observadas diferenças significativas entre os grupos com relação às fases do estresse e tipo de sintomatologia predominante. Em relação à ansiedade, não houve diferenças significativas entre os grupos infertilidade e controle quanto às medianas dos escores de estado (39,5 e 41,0, respectivamente) e traço (44,0 e 42,0, respectivamente) de ansiedade. No grupo infertilidade, os fatores de risco associados significativamente com estresse ou ansiedade foram: desconhecimento do fator causal, fase de investigação diagnóstica e ausência de filhos advindos de outros casamentos. Conclusões: as mulheres inférteis estão mais vulneráveis ao estresse, no entanto, são capazes de responder aos eventos estressores de forma adaptativa, sem comprometimentos mais sérios nas áreas física e psicológica.

PALAVRAS-CHAVE: Estresse; Ansiedade; Infertilidade feminina/psicologia; Reprodução; Questionários

\section{ABSTRACT}

Purpose: to assess the frequency of stress and anxiety levels in infertile women, in order to obtain data for specific psychological intervention. Methods: a cross-sectional study involving 152 infertile (mean age $30.3 \pm 5.4$ years), and 150 healthy control women (25.7 \pm 7.9 years). All patients were evaluated with the Lipp's Inventory of Stress Symptoms and the State-Trait Anxiety Inventory. Considered dependent variables were: stress frequency and anxiety scores (state and trait). Statistical analysis was performed by the $\chi^{2}$ and Mann-Whitney tests, and logistical regression to test associations between response variables and considered risk factors. Statistical significance was defined as $p<0.05$. Results: the stress was more frequent in the infertile group than in the control group ( 61.8 and $36.0 \%$, respectively); however, no significant differences were observed between groups in relation to stress phases and predominant symptoms. With respect to anxiety, there were no significant differences between infertile and control groups as to median state scores (39.5 and 41.0, respectively) and anxiety trait scores (44.0 and 42.0, respectively). Factors significantly associated with greater risk for high anxiety scores in the infertile group were: unawareness of the causal factor, diagnostic phase investigation, and lack of children from other marriages. Conclusions: it can be concluded that infertile women are more vulnerable to stress; however, they are capable of adapting themselves to stressful events without serious physical or psychological compromise.

KEYWORDS: Stress; Anxiety; Infertility, female/psychology; Reproduction; Questionnaires

Programa de Pós-graduação em Ciências da Saúde (PPGCSA) e Maternidade Escola Januário Cicco (MEJC) - Universidade Federal do Rio Grande do Norte - UFRN - Natal (RN) - Brasil.

1 Doutoranda do Programa de Pós-graduação em Ciências da Saúde (PPGCSA) - Universidade Federal do Rio Grande do Norte - UFRN - Natal (RN) Brasil.

2 Mestranda do Programa de Pós-graduação em Ciências da Saúde (PPGCSA) - Universidade Federal do Rio Grande do Norte - UFRN - Natal (RN) - Brasil.

3 Professor do Departamento de Tocoginecologia - Universidade Federal da Paraíba - UFPB - João Pessoa (PB) - Brasil.

4 Professor do Departamento de Morfologia e Orientador do PPGCSA - Centro de Biociências - Universidade Federal do Rio Grande do Norte - UFRN - Natal (RN) - Brasil.

Correspondência: Simone da Nóbrega Tomaz Moreira

Rua Minas Novas, 390, Condomínio Córdoba, casa 4 - Neópolis -59088-725 - Natal-RN

Apoio financeiro: CAPES

Recebido em: 26/4/2006

Aceito com modificaçōes em: 2/6/2006

Rev Bras Ginecol Obstet. 2006; 28(6): 358-64. 
Introdução

A infertilidade conjugal é definida como incapacidade de engravidar após um ano de atividades sexuais regulares sem o uso de qualquer método anticoncepcional ${ }^{1-3}$. Estima-se que cerca de 10 a $15 \%$ dos casais apresentem problemas para conceber. Os fatores causais masculinos e femininos contribuem isoladamente com cerca de $35 \%$ das causas, sendo o restante atribuído a fatores inexplicados (10\%) ou a associação de causas masculinas e femininas (20 a 30\%) ${ }^{4}$.

Pesquisadores estão divididos quanto à hipótese de que a infertilidade possa ter causas psicológicas (hipótese psicogênica) ou ser causa de uma variedade de sintomas psicológicos ${ }^{4,5}$. Até meados dos anos 1980, o modelo psicogênico foi dominante, porém estudos recentes salientam que a infertilidade pode ser a origem do estresse psicológico, pelo fato de os casais estarem expostos a grande quantidade de informações, diversos tipos de intervenções e novos tratamentos médicos, com o uso das tecnologias de reprodução assistida ${ }^{6}$.

Alguns estudos apontam que a utilização de protocolos diagnósticos extensos e tratamentos de longa duração pode precipitar sintomas psicológicos em 25 a $60 \%$ das pessoas inférteis, sendo de maior freqüência a ansiedade e a depressão, além da raiva, frustração, isolamento familiar/social e dificuldades sexuais ${ }^{2,4,7-9}$. A ansiedade costuma surgir em decorrência da natureza estressante dos tratamentos e do medo de não conceber, estando a depressão mais freqüentemente associada ao resultado negativo do tratamento ${ }^{10}$.

Os sintomas psicológicos advindos da infertilidade são complexos e influenciados por diversos fatores, como diferenças de gênero, causas e duração da infertilidade, estágio específico da investigação e do procedimento do tratamento, além da própria capacidade de adaptação ao problema e da motivação para ter filhos ${ }^{2,10}$.

A correlação entre estresse e infertilidade conjugal é estudada desde a década de 1950. Pesquisadores que avaliaram o nivel de estresse das mulheres submetidas à reprodução assistida constataram que aquelas que não lograram sucesso com o tratamento apresentaram maiores niveis de estresse do que as que conceberam ${ }^{3,6,11,12}$. Por outro lado, em outros estudos não se encontrou diferença em relação à média de estresse entre os indivíduos inférteis e férteis ${ }^{13,14}$. Nesse sentido, este trabalho se propõe a avaliar a presença de estresse e niveis de ansiedade em mulheres inférteis, procurando correlacioná-los com fatores de risco, de forma a obter subsídios para orientação psicológica adequada.
Métodos

Foi realizado estudo transversal, em que mulheres realizando tratamento para engravidar (grupo infertilidade, $n=152$ ) foram comparadas a um grupo controle $(n=150)$ constituido por mulheres da população geral, na mesma faixa etária, que não manifestavam dificuldade para conceber. O protocolo de pesquisa foi analisado e aprovado pelo Comitê de Ética em Pesquisa em Seres Humanos da Universidade Federal do Rio Grande do Norte, de acordo com o processo no 074/02.

Foram estabelecidos como critérios de inclusão comuns aos dois grupos: idade entre $18 \mathrm{e}$ 45 anos, capacidade cognitiva adequada para o entendimento dos propósitos da pesquisa, além da concordância em participar do estudo, após informação detalhada sobre os propósitos da pesquisa e assinatura do termo de consentimento livre e esclarecido. Foram excluidas mulheres que estavam vivenciando alguma situação estressante ou de muita ansiedade, como perdas de pessoas significativas na família, separação conjugal ou problemas na esfera profissional.

O grupo infertilidade foi constituído por mulheres apresentando tempo mínimo de infertilidade de 12 meses, recrutadas nos ambulatórios de Infertilidade Conjugal e Ginecologia Endócrina da Maternidade Escola Januário Cicco (MEJC), no período de agosto de 2002 a março de 2004. Para a formação do grupo controle, foram entrevistadas alunas do último ano do ensino médio das escolas da rede pública de Natal-RN (turno noturno), estudantes universitárias, além de funcionárias públicas e profissionais liberais.

A coleta de dados foi realizada por três entrevistadores capacitados e treinados na aplicação dos instrumentos. Para validação do processo de aplicação dos questionários, foi realizado estudo piloto inicial envolvendo 13 voluntárias, em que os questionários foram aplicados em duas ocasiões distintas, com intervalo mínimo de quatro semanas, sendo calculados coeficientes de variação individuais (desvio - padrão dividido pela média) para cada voluntária e escore considerado. A partir dessa análise inicial, os coeficientes de variação para os escores de estado e traço de ansiedade foram de 14,6 e 13,3\%, respectivamente.

A seqüência de aplicação dos questionários obedeceu à padronização em que as voluntárias foram inicialmente informadas sobre os propósitos da pesquisa e, após assinarem o termo de consentimento livre e esclarecido, foram convidadas a responder aos instrumentos de avaliação na seguinte ordem de aplicação: Ficha de Identifica- 
ção e Avaliação Médica; Inventário de Sintomas de Estresse de Lipp e Inventário de Ansiedade Traço-Estado. O primeiro instrumento constou de questionário elaborado de forma a abordar variáveis possivelmente implicadas como fatores de risco para a ocorrência de estresse e ansiedade, tais como: idade, tempo de infertilidade, fator de infertilidade, presença de filhos em outros casamentos, fase do acompanhamento (diagnóstica ou terapêutica), raça/etnia, religião, escolaridade e renda familiar.

Para avaliar a ocorrência de estresse foi utilizado o Inventário de Sintomas de Estresse de Lipp (ISSL) ${ }^{15}$, metodologia validada em 1994 por Lipp e Guevara e que tem sido utilizada em dezenas de pesquisas e trabalhos clínicos na área do estresse. Essa ferramenta avalia os sintomas físicos e psicológicos experimentados nas últimas 24 horas, na última semana e no último mês, permitindo estabelecer um diagnóstico preciso da ocorrência do estresse, fase em que se encontra (alerta, resistência, quase-exaustão e exaustão) e se a sintomatologia é predominante na área física ou psicológica. Na primeira fase, a do alerta, o organismo prepara-se para a reação de luta ou fuga, que é essencial para a preservação da vida. Se o estresse continua presente por tempo indeterminado, a fase de resistência inicia-se, pela qual o organismo tenta uma adaptação, devido à sua tendência de procurar a homeostase interna. Muitos dos sintomas iniciais desaparecem, dando lugar a uma sensação de desgaste e cansaço. Compreendida entre a fase da resistência e a da exaustão, encontra-se a fase da quase-exaustão, que se caracteriza por enfraquecimento da pessoa que não mais está conseguindo adaptar-se ou resistir ao fator estressor. Nessa fase, as doenças começam a surgir, porém, ainda não são tão graves como na fase de exaustão, na qual aparecem as doenças mais sérias e a pessoa, na maioria das vezes, não consegue trabalhar ou concentrar-se ${ }^{15}$. A aplicação do ISSL é prática e fácil, levando cerca de dez minutos para ser administrado e não sendo necessário que o respondente seja alfabetizado, visto que os itens podem ser lidos pelo entrevistador para a pessoa. A sua correção e interpretação devem ser realizadas por psicólogo, de acordo com as diretrizes do Conselho Federal de Psicologia quanto ao uso de testes.

Para mensurar as variáveis "traço de ansiedade" e "estado de ansiedade" foi utilizado o Inventário de Ansiedade Traço-Estado (IDATE), elaborado por Spielberger et al. em $1970^{16}$. Essa escala foi validada e traduzida para a língua portuguesa por Biaggio e Natalício em 1979 e revelou-se como tendo uma alta confiabilidade e bom constructo de validade. Esse instrumento é composto por duas escalas distintas, que visam a medir dois conceitos diferentes: estado e traço de ansiedade.

O estado de ansiedade é conceituado como estado emocional transitório do organismo, ao passo que o traço de ansiedade refere-se a diferenças individuais relativamente estáveis de propensão à ansiedade, isto é, a diferenças na tendência de reagir a situações percebidas como ameaçadoras. As pessoas que diferem em traço de ansiedade mostrarão ou não diferenças correspondentes em estado de ansiedade, na dependência do grau em que a situação específica é percebida como perigosa ou ameaçadora, o que, por sua vez, é fortemente influenciado por experiências passadas do indivíduo $^{16}$. O traço de ansiedade ou ansiedade não situacional pode ser um estado de início recente ou uma característica persistente da personalidade do indivíduo ${ }^{17}$.

Ambas escalas consistem de 20 afirmações cada. Os escores possíveis para ambas escalas variam de 20 a 80 pontos, sendo que escores altos indicam alto nivel de ansiedade e escores baixos, baixo nível de ansiedade.

Uma dificuldade metodológica nos estudos envolvendo o IDATE diz respeito à necessidade da utilização de parâmetros específicos para cada população estudada. Em virtude da inexistência de parâmetros de normalidade provenientes de estudos realizados em população semelhante, foram empregados pontos de corte estabelecidos na literatura, por outros pesquisadores que utilizaram essa metodologia. Para esse fim, foram considerados parâmetros previamente estabelecidos por Rosa $^{17}$, em que as categorias de ansiedade variam da intensidade "baixa" (escores de 0 a 29), "médiabaixa" (30 a 39), "média" (40), "média-alta" (41 a 50) e "elevada" (51 a 80). Conforme corroborado por outros autores, o ponto de corte empregado no presente trabalho para atribuição da categoria de "alto risco" para ansiedade foi de $40^{18}$.

Para cálculo da estimativa da amostra, os questionários foram inicialmente aplicados a uma amostra - piloto de 30 mulheres do grupo controle, visando à mensuração da freqüência de estresse e dos escores médios de ansiedade (traço e estado) nessa amostra. A partir desses dados, foram calculadas estimativas amostrais suficientes para demonstrar aumento de, no mínimo, 20\% no grupo infertilidade, em relação à freqüência de estresse e escores médios de ansiedade. Para tal fim, foi utilizado o software estatístico GraphPad StatMate, version 1.01i, (GraphPad Software, San Diego, CA, USA), adotando-se alfa de $5 \%$ e poder estatístico de 80\%. Em virtude do cálculo de um tamanho amostral para cada variável testada, 
optou-se por adotar aquele com maior número de sujeitos, resultando numa estimativa amostral de 150 voluntárias por grupo.

Os resultados são apresentados em tabelas, onde as variáveis são expressas em média \pm desviopadrão e mediana (percentil25-percenti175) para variáveis quantitativas e em freqüências simples para variáveis qualitativas. Para verificação da normalidade dos valores amostrais foi utilizado o teste não paramétrico de Kolmogorov-Smirnov. Para comparações de resultados intergrupos foram utilizados os testes $t$ de Student (para variáveis com distribuição normal) e teste de Mann-Whitney (para variáveis com distribuição não paramétrica). As correlações entre variáveis foram avaliadas pelo teste de correlação de Pearson.

As comparações intergrupos das freqüências das diversas categorias consideradas no estudo foram realizadas pelo teste $\chi^{2}$ (análise bivariada) e por metodologia de regressão logística, com cálculo da odds ratio e intervalos de confiança (IC 95\%). Para cada variável-resposta, os modelos de regressão logística foram obtidos a partir de um processo de seleção construído pela inserção progressiva das variáveis, denominado de FORWARD method, com base na estatística de Wald ${ }^{19}$. Para categorização das variáveis dependentes "estado" e "traço" de ansiedade, foi empregado o ponto de corte de 40 para atribuição de baixo e alto risco de ansiedade. O nível de significância considerado em todos tratamentos estatísticos realizados foi de $5 \%$. As análises foram implementadas utilizandose os softwares GraphPad Prism, version 3.00 for Windows (GraphPad Software, San Diego, CA, USA) e Statistical Package for the Social Sciences - SPSS (version 10.0 for Windows).

\section{Resultados}

Caracterizando-se a amostra estudada quanto à presença de estresse, de acordo com o ISSL, observou-se que $61,8 \%$ das mulheres do grupo infertilidade e 36,0\% das mulheres do grupo controle apresentaram estresse, havendo diferenças significantes entre essas freqüências relativas (Tabela 1). De acordo com esses resultados, a chance de uma pessoa vir a apresentar estresse é 2,9 vezes maior se ela pertence ao grupo das mulheres inférteis ( $\mathrm{IC}=1,8-4,6)$.

Conforme mostra a Tabela 1 , não foram observadas diferenças significativas entre os grupos, com relação às fases do estresse. A fase predominante nos dois grupos foi a de "resistência" e houve predominância dos sintomas psicológicos sobre os sintomas físicos, em ambos grupos, sem diferença significativa (dados não apresentados).

Analisando-se exclusivamente os fatores de risco para ocorrência de estresse no grupo infertilidade, o modelo de regressão logística apresentado na Tabela 2 mostra que alguns fatores estiveram

Tabela 1 - Freqüências e fases de estresse entre os grupos estudados, de acordo com o Inventário de Sintomas de Estresse de Lipp.

\begin{tabular}{lllll}
\hline \multirow{2}{*}{ Estresse } & \multicolumn{2}{l}{ Grupo infertilidade } & \multicolumn{2}{c}{ Grupo controle } \\
& $\mathbf{n}$ & $\%$ & $\mathbf{n}$ & $\%$ \\
\hline Presente & 94 & $61,8^{*}$ & 54 & $36,0^{*}$ \\
Fase 1- Alerta & 4 & 4,3 & 1 & 1,9 \\
Fase 2- Resistência & 78 & 83,0 & 49 & 90,7 \\
Fase 3- Quase-exaustão & 10 & 10,6 & 2 & 3,7 \\
Fase 4- Exaustão & 2 & 2,1 & 2 & 3,7 \\
Ausente & 58 & 38,2 & 96 & 64,0 \\
Total & 152 & 100 & 150 & 100 \\
\hline
\end{tabular}

Nota: * $p<0,001$, teste $\chi^{2}$ relação à freqüência de estresse entre os grupos. Não foram observadas diferenças significativas quanto à distribuição das fases do estresse ( $p$ > $0,05)$ entre os dois grupos avaliados.

Tabela 2 - Associação entre fatores de risco potencialmente estressores e a ocorrência ou não de estresse em mulheres inférteis, utilizando o modelo de regressão logística.

\begin{tabular}{|c|c|c|c|c|c|c|c|}
\hline & \multicolumn{2}{|c|}{ Estresse presente } & \multicolumn{2}{|c|}{ Estresse ausente } & \multirow{2}{*}{$p$} & \multirow{2}{*}{ OR } & \multirow{2}{*}{ IC $95 \%$} \\
\hline & $\mathrm{n}$ & $\%$ & $\mathrm{n}$ & $\%$ & & & \\
\hline \multicolumn{8}{|l|}{ Filhos em outros casamentos } \\
\hline Sim (CatRef) & 13 & 13,8 & 21 & 36,2 & & & \\
\hline Não & 81 & 86,2 & 37 & 63,8 & 0,006 & 3,7 & {$[1,5-9,5]$} \\
\hline \multicolumn{8}{|l|}{ Fator de infertilidade } \\
\hline Não esclarecido (CatRef) & 36 & 38,3 & 10 & 17,2 & & & \\
\hline Masculino & 6 & 6,4 & 1 & 1,7 & 0,663 & 1,7 & {$[0,2-16,8]$} \\
\hline Feminino & 49 & 52,1 & 46 & 79,3 & 0,008 & 0,3 & {$[0,1-0,7]$} \\
\hline Misto & 3 & 3,2 & 1 & 1,7 & 0,662 & 1,9 & {$[0,1-23,6$} \\
\hline \multicolumn{8}{|l|}{ Fase do acompanhamento } \\
\hline Investigação diagnóstica (CatRef) & 73 & 77,7 & 28 & 48,3 & & & \\
\hline Tratamento & 21 & 22,3 & 30 & 51,7 & $<0,001$ & 0,2 & {$[0,1-0,4]$} \\
\hline
\end{tabular}

Nota: na tabela são apresentadas apenas variáveis com associação significativa; OR = odds ratio; IC = intervalo de confiança; CatRef = categoria de referência 
significativamente associados com o estresse, tais como: ausência de filhos em outros casamentos, desconhecimento da causa de infertilidade e fase de investigação diagnóstica. Associando-se a variável "estresse" com os escores de ansiedade obtidos por meio do IDATE, evidenciou-se que as mulheres com menor traço de ansiedade (escores inferiores a 40) apresentaram menor propensão a sofrer de estresse (odds ratio $=0,3-$ IC 95\% = 0,2-0,5). A partir desses dados, pode-se inferir que aquelas com escores de traço de ansiedade mais baixos apresentam aproximadamente um terço da chance de apresentar estresse, em relação às mulheres com escores mais elevados.

Em relação à ansiedade, não houve diferenças significativas entre os grupos infertilidade e controle quanto às medianas dos escores de estado (39,5 e 41,0, respectivamente) e traço (44,0 e 42,0, respectivamente) de ansiedade. Nas comparações intragrupos, foram observadas diferenças significativas entre os escores do estado e traço de ansiedade apenas no grupo infertilidade, em que os escores do traço foram significativamente maiores do que os escores do estado de ansiedade (Figura 1). Relacionando-se os valores do estado e traço de ansiedade com a presença de estresse (Figura 2), observou-se tendência de elevação dos escores de estado de ansiedade conforme progridem as fases do estresse, no grupo constituído por mulheres inférteis, mas sem diferenças significantes.

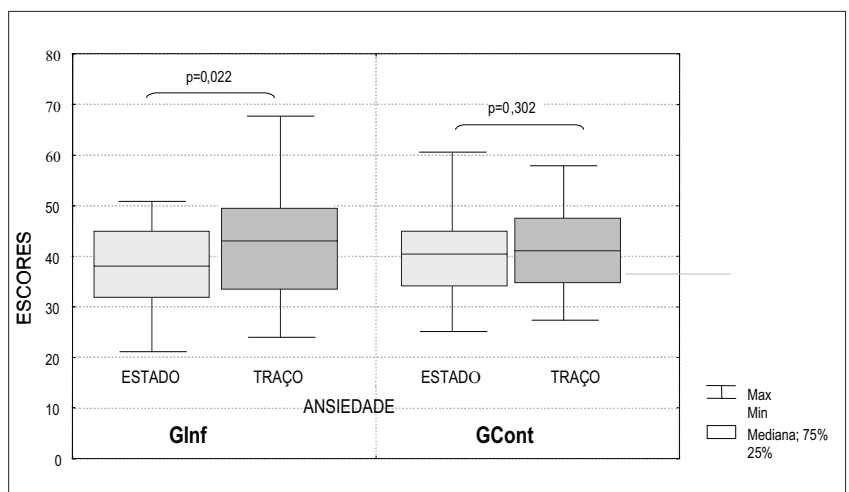

Figura 1 - Representação gráfica dos escores de ansiedade (estado e traço), nos grupos infertilidade (GInf) e controle (GCont), obtidos a partir do IDATE.

\section{Discussão}

Em relação à presença de estresse, avaliada pelo ISSL, observamos maior freqüência de estresse nas mulheres inférteis do que naquelas do grupo controle. Ao comparar esses dados com os da literatura, percebe-se que os pesquisadores que avaliaram a presença do estresse nas mulheres inférteis têm encontrado resultados controversos: alguns trabalhos apontam que existe relação entre a infertilidade e o estresse $\mathrm{e}^{3,6,11,12}$, ao passo que outros negam tal relação ${ }^{13,14}$. Isso tem sido explicado pelas inconsistências metodológicas encontradas nos diversos trabalhos. Alguns estudos afirmam que se deveria considerar a duração da infertilidade

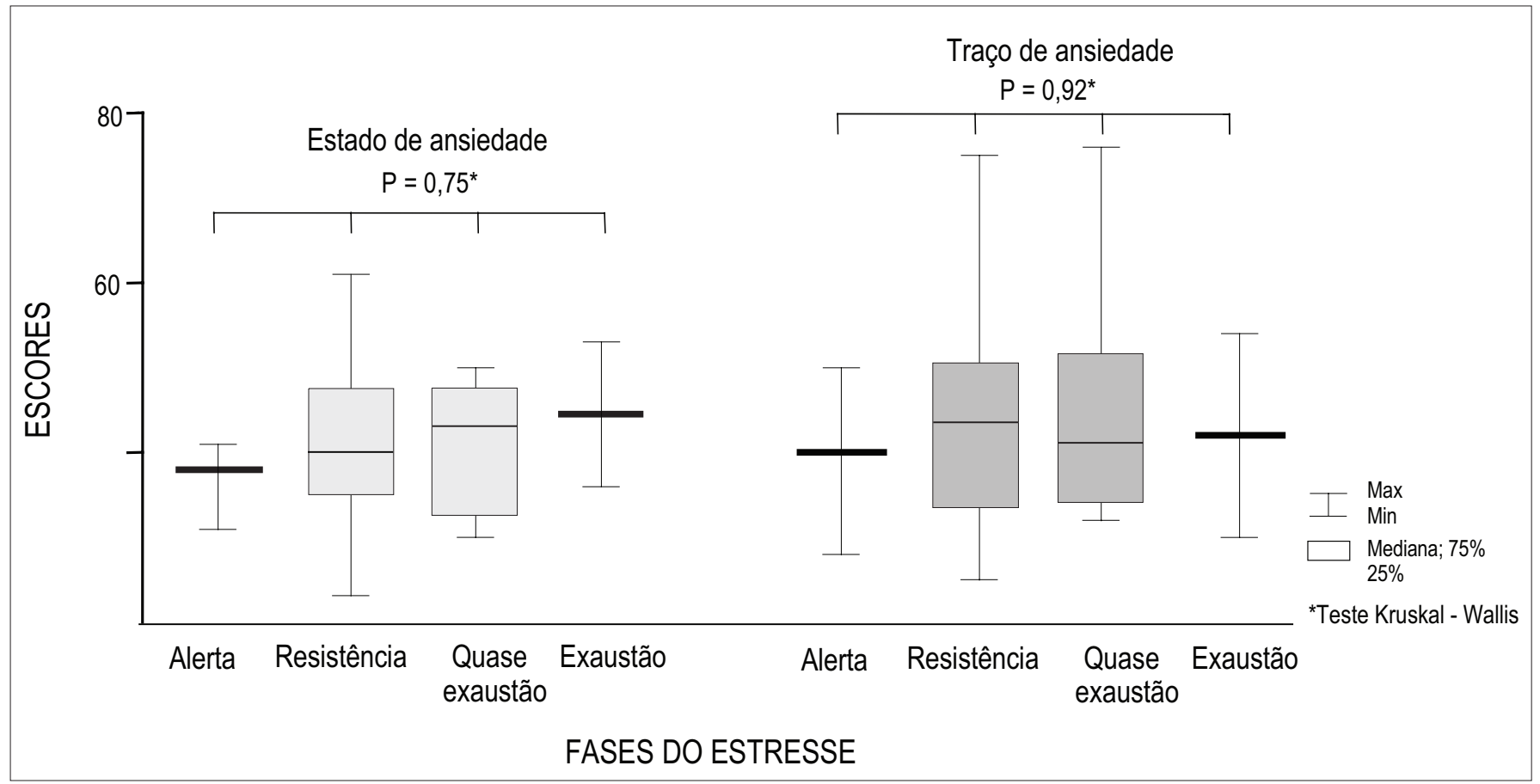

Figura 2 - Relação entre o estado e traço de ansiedade e as fases do estresse no grupo infertilidade. 
e os procedimentos terapêticos a que os casais estão submetidos. Outros estudos referem-se ao pequeno tamanho das amostras e à falta de conhecimento dos pesquisadores de técnicas estatísticas apropriadas para a análise dos resultados ${ }^{8}$. Além disso, a inexistência de instrumentos específicos que possam avaliar o estresse dos casais inférteis ${ }^{14}$ é outro aspecto que deve ser considerado.

Os resultados obtidos mostraram que das 94 mulheres inférteis que apresentaram estresse, 78 $(83 \%)$ se encontravam na fase de resistência, na qual sintomas mais freqüentes foram: mal-estar, sensação de desgaste físico, cansaço, sensibilidade emotiva excessiva e irritabilidade. Nessa fase, o indivíduo pode aprender a lidar com suas tensões, eliminando os seus sintomas e prevenindo o surgimento de comprometimentos físicos ou psicológicos advindos do estresse, como é o caso da ansiedade. Entretanto, mesmo nessa fase, há necessidade do apoio e aconselhamento psicológico, com o objetivo de ajudar as mulheres a desenvolver estratégias adaptativas diante dos sintomas apresentado ${ }^{20}$. A fase de quase-exautão compreendeu 10 mulheres do grupo infertilidade, o que sugere que essas mulheres já começaram a apresentar comprometimentos físicos ou psicológicos, porém ainda não tão graves como na fase de exaustão, que envolveu apenas duas mulheres. Nesse estágio, além do surgimento de doenças como hipertensão arterial, problemas dermatológicos prolongados, sintomas gástricos, entre outros, elas podem apresentar os seguintes sintomas psicológicos: depressão, raiva, ansiedade, angústia, apatia e hipersensibilidade emotiva.

O fato observado da maior ocorrência de estresse entre mulheres que ainda não vivenciaram a experiência da gravidez e maternidade levanta questionamentos sobre possiveis fatores capazes de explicar o fenômeno. Sabe-se que essas mulheres se sentem pressionadas pelo valor cultural vigente, em que a maternidade é vista como destino biológico e está associada à realização feminina. Ainda em relação aos fatores de risco, os resultados desta pesquisa apontam que a investigação diagnóstica e o desconhecimento da causa de infertilidade provocam maior ocorrência de estresse. Isso pode ser explicado pelo fato de que, nessa fase, as mulheres sentem-se vulneráveis, submetem-se a exames invasivos e, muitas vezes, não recebem apoio do companheiro, que adia a sua ida ao médico, rejeitando a hipótese de que também possa apresentar algum fator masculino de infertilidade, motivado por fatores culturais que relacionam infertilidade com potência sexual.

Em relação à avaliação da ansiedade, por meio do Inventário de Ansiedade Traço-Estado, os resultados mostraram que os escores médios observados para traço e estado de ansiedade nas mulheres inférteis não diferiram significativamente daqueles obtidos no grupo controle. Analisando especificamente o grupo infertilidade, a ocorrência de escores mais elevados para o traço de ansiedade, em relação ao estado, pode sugerir que as mulheres inférteis apresentam tendência de reagir com maior intensidade de ansiedade diante de situações adversas. Entretanto, deve-se levar em consideração o fato de que o conceito de ansiedade não envolve um construto unitário, podendo ser generalizado ou focado em situações específicas. Saliente-se o fato de que ainda não existe diferenciação precisa entre traço e estado de ansiedade, devido à alta correlação de determinados itens que compõem as escalas ${ }^{17}$.

Relacionando-se os valores do estado de ansiedade e a presença de estresse, percebeu-se tendência de elevação dos escores do estado de ansiedade conforme progridem as fases do estresse, no entanto, sem significância do ponto de vista estatístico. Tal fato pode ser atribuído ao número reduzido de mulheres nas fases de quase-exaustão e exaustão, que certamente comprometeu a análise estatística. Essa tendência tem sido confirmada por diversos autores, que atestam a maior ocorrência de ansiedade nas fases mais avançadas do estresse, caracterizando o chamado distresse psicológico ${ }^{15}$.

Vários pesquisadores utilizaram o Inventário de Ansiedade Traço-Estado (IDATE) para avaliar em que medida essa variável psicológica pode influenciar a função reprodutiva. Em mulheres submetidas à inseminação artificial, os resultados apontam que quanto maior o nivel de ansiedade, menor a chance de gravide $z^{11,12}$. Os resultados do presente estudo reforçam o trabalho de Milad et al. ${ }^{13}$, que não encontraram diferenças significativas nos niveis de ansiedade entre mulheres que conceberam e aquelas que não engravidaram, após procedimento de fertilização in vitro. Pesquisadores que avaliaram a ansiedade e a depressão do casal submetido ao tratamento de infertilidade concluíram que a prevalência de distúrbios emocionais foi baixa, contudo, as mulheres apresentaram maior escore de ansiedade em relação aos homen ${ }^{14}$.

Em conclusão, os resultados de nosso trabalho apontam que as mulheres inférteis estão mais vulneráveis ao estresse, principalmente aquelas que nunca tiveram filhos, apresentando maior tendência de reagir a situações ameaçadoras com intensidade mais elevada de ansiedade. Esses achados nos permitem sugerir que as intervenções psicológicas nos serviços de reprodução humana deveriam ocorrer em dois níveis: a avaliação psicológica no início do tratamento, com o objetivo de identificar de forma preventiva as mulheres que apresentam problemas de ajustamento emocional, 
possibilitando assim, o encaminhamento ao atendimento psicológico específico e o apoio psicológico durante e após cada intervenção médica, para propiciar a essas mulheres as condições de valorizar a experiência vivida, de forma que possam atingir maturidade emocional.

\section{Referências}

1. Van Balen F, Trimbos-Kemper T, Verdurmen J. Perception of diagnosis and openness of patients about infertility. Patient Educ Couns. 1996; 28(3):247-52.

2. Gerrity DA. A biopsychosocial theory of infertility. Fam J. 2001; 9(2):151-8.

3. Salvatore P, Gariboldi S, Offidani A, Coppola F, Amore M, Maggini C. Psychopathology, personality, and marital relationship in patients undergoing in vitro fertilization procedures. Fertil Steril. 2001;75(6):1119-25.

4. Bell JS. Psychological problems among patients attending an infertility clinic. J Psychosom Res. 1981;25(1):1-3.

5. Gulseren L, Cetinay P, Tokatlioglu B, Sarikaya OO, Gulseren S, Kurt S. Depression and anxiety levels in infertile Turkish women. J Reprod Med. 2006;51(5):421-6.

6. Facchinetti F, Matteo ML, Artini GP, Volpe A, Genazzani AR. An increased vulnerability to stress is associated with a poor outcome of in vitro fertilization-embryo tranfer treatment. Fertil Steril. 1997;67(2):309-14.

7. Dyer SJ, Abrahams N, Mokoena NE, Lombard CJ, van der Spuy ZM. Psychological distress among women suffering from couple infertility in South Africa: a quantitative assessment. Hum Reprod. 2005;20(7):1938-43.

8. Greil AL. Infertility and psychological distress: a critical review of the literature. Soc Sci Med. 1997;45(11):1679-704.

9. Daniluk JC. "If we had it to do over again...": Couples' reflections on their experiences of infertility treatments. Fam J. 2001;9(2):122-33.

10.Ozkan M, Baysal B. Emotional distress of infertile women in Turkey. Clin Exp Obstet Gynecol. 2006;33(1):44-6.

\section{Agradecimentos}

Ao Prof. Dr. Ângelo Giuseppe Roncalli da Costa e a João Evangelista Pereira pelas contribuições na análise estatística. A Franklin de Freitas Tertulino e Cleyton Amaral Nogueira e Silva, pelo auxílio na fase de coleta dos dados.

11.Klonoff-Cohen H, Chu E, Natarajan L, Sieber W. A prospective study of stress among women undergoing in vitro fertilization or gamete intrafallopian transfer. Fertil Steril. 2001;76(4):675-87.

12. Gallinelli A, Roncaglia R, Matteo ML, Ciaccio I, Volpe A, Facchinetti F. Immunological changes and stress are associated with different implantation rates in patients undergoing in vitro fertilization-embryo transfer. Fertil Steril. 2001;76(1):85-91.

13. Milad MP, Klock SC, Moses S, Chatterton R. Stress and anxiety do not result in pregnancy wastage. Hum Reprod. 1998;13(8):2296-300.

14.Anderson KM, Sharpe M, Rattray A, Irvine DS. Distress and concerns in couples referred to a specialist infertility clinic. J Psychosom Res. 2003;54(1):353-5.

15.Lipp MN. Manual do inventário de sintomas de stress de Lipp. São Paulo: Casa do Psicólogo; 2000. p. 9-26.

16. Spielberger CD, Gorsuch RL, Lushene RE. Inventário de ansiedade traço-estado. Manual de psicologia aplicada. Rio de Janeiro: CEPA; 1979.

17.Rosa JL. Inventário de ansiedade traço - estado: características psicométricas. Estud Psicol. 1993;10(2):81-92.

18. Matos MIR, Aranha LS, Faria AN, Ferreira SRG, Bacaltchuck J, Zanella MT. Binge eating disorder, anxiety, depression and body image in grade III obesity patients. Rev Bras Psiquiatr. 2002;24(4):165-9.

19.Pereira JCR. Análise de dados qualitativos: estratégias metodológicas para as ciências da saúde, humanas e sociais. 3a ed. São Paulo: Ed Universidade de São Paulo; 2001.

20.de Liz TM, Strauss B. Differential efficacy of group and individual/couple psychotherapy with infertile patients. Hum Reprod. 2005;20(5):1324-32. 